\title{
Multilayer film stability
}

\author{
N. Sridhar \\ Department of Materials Science and Engineering, University of Michigan, Ann Arbor, Michigan 48109 \\ J. M. Rickman \\ Department of Materials Science and Engineering, Lehigh University, Bethlehem, Pennsylvania 18015 \\ D. J. Srolovitz ${ }^{\text {a) }}$ \\ Department of Materials Science and Engineering, University of Michigan, Ann Arbor, Michigan 48109
}

(Received 14 March 1997; accepted for publication 21 July 1997)

\begin{abstract}
We apply a linear stability analysis to examine the effect of misfit stress on the interface diffusion controlled morphological stability of multilayer microstructures. The stresses could be the result of misfit strains between the individual film layers and/or between film and substrate. We find that misfit between the layers in the film can destabilize the multilayer structure in cases where the thinner layer is elastically stiffer than the thicker layer. The rate at which these instabilities develop increase with increasing misfit and decreasing interfacial energy. Even when there is no misfit between layers, the misfit between the multilayer film and substrate can destabilize the interfaces. This type of instability occurs whether the thinner layers are stiffer or more compliant than the thicker ones. By appropriate choice of the elastic moduli mismatch between layers and relative layer thicknesses, the presence of an interlayer misfit can suppress the instability caused by the substrate misfit. We present stability diagrams that can be used to design stable, multilayer films using all of the degrees of freedom commonly available in multilayer film deposition. (C) 1997 American Institute of Physics. [S0021-8979(97)02421-3]
\end{abstract}

\section{INTRODUCTION}

Multilayer thin films are excellent candidate materials for novel electronic and photonic device applications. ${ }^{1}$ For example, short period superlattices of InAs/GaAs and $\mathrm{InP} /$ $\mathrm{GaP}$ are now being exploited as nanometer scale quantum well and quantum wire heterostructures. ${ }^{2}$ These structures exhibit superior laser diode device properties, including lower threshold current, higher modulation bandwidth, and greater temperature stability as compared with bulk heterostructure devices. ${ }^{3}$ Another example is InGaAsP-based multiple quantum well structures, with alternating compressive and tensile strained layers, which are promising for optoelectronic device applications, such as light sources and detectors in optical fiber communications systems. ${ }^{4}$ In addition to electronic applications, multilayer films find use as x-ray mirrors ${ }^{5}$ and as coatings that are both strong and tough. ${ }^{6}$

An important consideration, which determines the technological success of these materials, is their microstructural stability. For instance, alternating compressive and tensile strained layers in InGaAsP-based multiple quantum well structures are often designed to be strain balanced. However, lateral variations in surface morphology and alloy composition have been observed which leads to degradation in optical properties. ${ }^{7}$ Lateral strain modulations, surface undulations, and interface undulations have also been observed in strained heterostructures including III-V compounds as well as in $\mathrm{SiGe} / \mathrm{Si}^{8}{ }^{8}$ Dramatic thickness modulations have also been observed in strained GaInAsP/(001)InP multilayers grown by gas source molecular beam epitaxy. ${ }^{9}$ These experimental observations suggest that instabilities occur both during and postgrowth. Postgrowth instabilities include morpho-

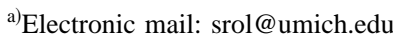

logical instabilities of the interfaces between the layers (primarily for immiscible layers) or compositional modulations in miscible multilayer films. In this article, we examine postgrowth morphological instabilities in immiscible multilayer microstructures. We will identify the factors that control the stability of these films and estimate rates at which these morphological instabilities develop.

The stability of lattice-mismatched thin films has received a great deal of attention theoretically. A recently proposed mechanism suggests that elastic stresses induce morphological instabilities, ${ }^{10-12}$ which can lead to the formation of islands ${ }^{13}$ nonplanar surfaces ${ }^{8}$ or, in some cases, the formation of deep, cusplike morphologies. ${ }^{14,15}$ Such morphologies can then provide sources for the nucleation of stressrelieving dislocations. ${ }^{15}$ The wavelength of this type of instability is set by the competition between the stabilizing influence of the surface energy and the destabilizing influence of the misfit-induced elastic strain energy. The analyses described may be/have been applied to the stability of single layer films. The case of multilayer films is significantly more complicated because it must include the effects of the relative magnitudes of the interlayer misfit strain and that imposed by the substrate in addition to the material (e.g., interfacial energies, elastic constants of multiple phases) and geometrical (i.e., layer thicknesses) parameters.

Recently, we examined the stability of lamellar, eutectic microstructures. ${ }^{16}$ The stability of such microstructures may be directly mapped onto the problem of multilayer films. In this article, we employ the results from Ref. 16 to the specific case of multilayer films. In Sec. II we examine morphological stability by calculating the chemical potential along the interface. The chemical potential is incorporated in a kinetic model in order to calculate the growth rate of a pertur- 

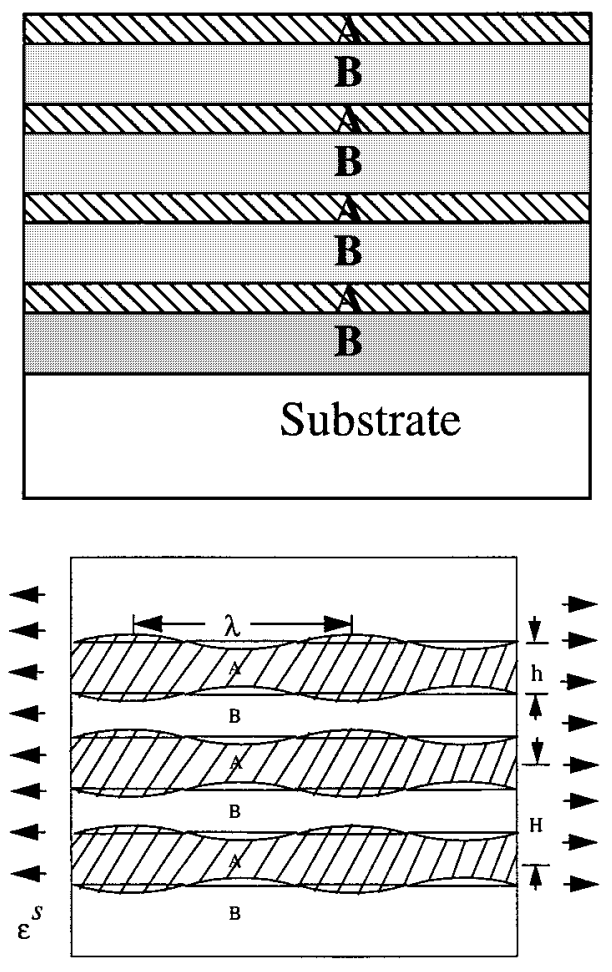

FIG. 1. (a) Schematic illustration of a periodic multilayer A/B film on a substrate. (b) A view of the multilayer film structure far from the free surface and the substrate, considered here. The interfaces are perturbed by the profile described in Eq. (1).

bation to the interface shape. In Sec. III the influence of material properties on the growth rate is systematically examined. We also present stability diagrams that identify combinations of material and geometrical properties required to maintain a flat (i.e., stable) interface.

\section{PHYSICAL MODEL AND LINEAR STABILITY ANALYSIS}

Consider a multilayer film, consisting of a very large number of layers, grown on a substrate, as shown in Fig. 1(a). We assume that the multilayer consists of two types of layers (A and B) and that the relative thicknesses of these layers remain constant through the entire film thickness. The overall wavelength of the multilayer is defined as $\mathrm{H}$ and the thickness of the A and B layers are $h$ and $H-h$, respectively. The two types of layers are assumed to be isotropic, linear elastic media and can have different elastic constants. These layers may be misfitting with respect to one another. In the present analysis, we assume that the misfit of the A layers with respect to the $\mathrm{B}$ layers are described by an eigenstrain that is isotropic with components $\epsilon_{i j}^{*}=\epsilon^{*} \delta_{i j}$, which corresponds to a uniform volume dilatation. The multilayer film may also be misfitting with respect to the substrate. If the multilayer film were strain balanced with respect to the substrate (i.e., it would not deform if we separated the film from the substrate), we would define this misfit strain with respect to the substrate, $\epsilon^{s}=0$. In the general cases, this misfit strain tensor can take on any set of values. In the present case, we limit consideration to the special case of a uniaxial substrate misfit strain $\epsilon_{x x}=\epsilon^{s}$ in order to keep the problem pseudotwo-dimensional. In the elastic analysis of this multilayer film on a substrate, we explicitly assume that all of the interfaces are elastically coherent, in the sense that all tractions and displacements are continuous across the interfaces.

In examining the stability of the interfacial morphology, we explicitly consider the case in which the two layers consist of materials which are mutually immiscible and that the only transport mechanism that operates is interfacial diffusion. We further assume that the interface diffusivities of the two materials are equal, and therefore there is no net matter accumulation/depletion along the interface. In addition, the interfaces are assumed to be coherent and characterized by an isotropic interface energy. We also ignore surface stress effects. Since the film consists of a very large number of layers, we will focus the analysis on those layers which are not too close to either the free surface or the substrate. In this way, the film geometry shown in Fig. 1(a) can be simplified to the perfectly periodic geometry shown in Fig. 1(b). While these assumptions greatly simplify the analysis, there are many cases in real materials where each assumption is violated. Therefore, care must be exercised in specific applications.

We examine the stability of the interfaces by considering the effects of a small perturbation to the nominally flat interface profile:

$$
y_{i}(x)= \pm[(h / 2)+\delta \cos (k x)],
$$

where $\delta$ is the amplitude of the perturbation, the perturbation wave number $k=2 \pi / \lambda$, and $\lambda$ is the wavelength of the perturbation. We assume that $\delta k \ll 1$, which implies that the interface has a small slope everywhere. The chemical potential $(\mu)$ along the perturbed interface taking into account both curvature and elastic effects is ${ }^{17,18}$

$$
\mu-\mu_{0}=\Omega\left(\gamma \kappa+[W]_{-}^{+}-\mathbf{T} \cdot\left[\frac{\partial \mathbf{u}}{\partial n}\right]_{-}^{+}\right),
$$

where $\mu_{0}$ is the chemical potential of a flat interface, $\Omega$ is the atomic volume, $\gamma$ is the isotropic interface energy, $\kappa$ is the interface curvature, $W$ is the strain energy density, $\mathbf{T}$ is the interfacial traction vector, $\partial \mathbf{u} / \partial n$ is the derivative of the total displacement field with respect to the direction normal to the interface $n$, and the notation $[q]_{-}^{+}$denotes the jump (or difference) in the quantity $q$ across the interface. Note that the interface normal, traction, etc., are all defined with respect to the actual (nonflat) interface profile. The + sign represents the B layer side of the interface in this notation. If the chemical potential along the interface is not spatially constant, matter transport from regions of high to low chemical potential will occur. The elastic fields for the layered geometry considered here were presented in detail in Ref. 16.

Once the chemical potential is known, the force acting on atoms along the interface is simply $F=-\partial \mu / \partial s$, where $s$ is the arc length along the interface. Using Fick's first law, the atomic flux $J$ along the interface is $J=M F$, where the mobility $M=D_{i} \eta_{i} / \Omega k_{B} T, D_{i}$ is the interface diffusivity, $\eta_{i}$ is the effective thickness of the interface layer participating in the interface diffusion process, and $k_{B} T$ is the thermal energy at temperature $T$. Employing the requirement of con- 
servation of matter, the interface velocity in the normal direction (the normal pointing away from the interface into phase B) may be found in terms of the divergence of the material fluxes along the interface:

$$
V_{i}=-\Omega \frac{\partial J}{\partial s}=M \Omega^{2} \frac{\partial^{2} \mu}{\partial s^{2}} .
$$

Inserting the initial interface profile [Eq. (1)] into Eq. (3) yields an equation for the initial evolution of the interface profile

$$
V_{i}=\mathbf{n} \cdot \frac{\partial \mathbf{X}}{\partial t} \approx \frac{\partial \delta}{\partial t} \cos (k x),
$$

where $\mathbf{n}$ is the unit vector along the interface normal, $\mathbf{X}$ is a vector describing the position of the interface, and $t$ is the time. Upon equating the right-hand sides of Eqs. (3) and (4) and substituting in for $\mu$, we arrive at a differential equation that governs the time evolution of the interfacial amplitude, $\delta$. The solution to this differential equation can be conveniently written in the general form

$$
\delta(\tau)=\delta(0) \exp (\phi \tau)
$$

where the dimensionless time $\tau=t \Omega^{2} M \gamma / h^{4}$ and the dimensionless growth rate $\phi=G h^{4} / \Omega^{2} M \gamma$, where $G$ is the unnormalized growth rate. Thus, if $\phi$ is positive, the perturbation grows and, consequently, the flat interface is unstable. In the present case, the growth rate, $\phi$, can be rewritten as

$$
\phi=\phi_{c}+\phi_{e}=-(k h)^{4}+\phi_{e},
$$

where $\phi_{c}$ and $\phi_{e}$ are the curvature and elastic contributions to the growth rate, respectively. As seen from Eq. (6), the curvature contribution to the growth rate is always negative and, therefore, the flat interface is stable in the absence of elastic effects. Since $\phi_{e}$ is a complicated dimensionless function of the material and geometrical parameters, we restrict ourselves to a graphical description of the dependence of the growth rate on these variables in the next section.

\section{RESULTS AND DISCUSSION}

\section{A. Interlayer misfit strain}

We first focus on a multilayer in which the A and B layers have a dilatational misfit $\epsilon^{*}$ with respect to each other and that the multilayer film is strain balanced with respect to the substrate (i.e., $\epsilon^{s}=0$ ). Such misfit strains may be the result of heteroepitaxy or from such effects as thermal expansion mismatch or phase transformations. The relative importance of capillarity and elastic effects on the growth rate can be captured in the form of two-dimensionless control parameters: $\theta=\gamma / E_{A} h\left(\epsilon^{*}\right)^{2}$ and $\alpha=\left(E_{A}-E_{B}\right) /\left(E_{A}+E_{B}\right)$, where $E_{A}$ and $E_{B}$ are the moduli of the two layers, respectively. We set the Poisson ratios $\nu_{A}=\nu_{B}=1 / 3$.

\section{Buried layer case}

We first consider the special case of a thin layer of A within a relatively thick film of B, i.e., a buried layer. Using the elastic fields in Ref. 16, we determine the chemical potential everywhere and the perturbation growth rate $\phi$. Figure 2 shows the variation of $\phi$ with the dimensionless wave

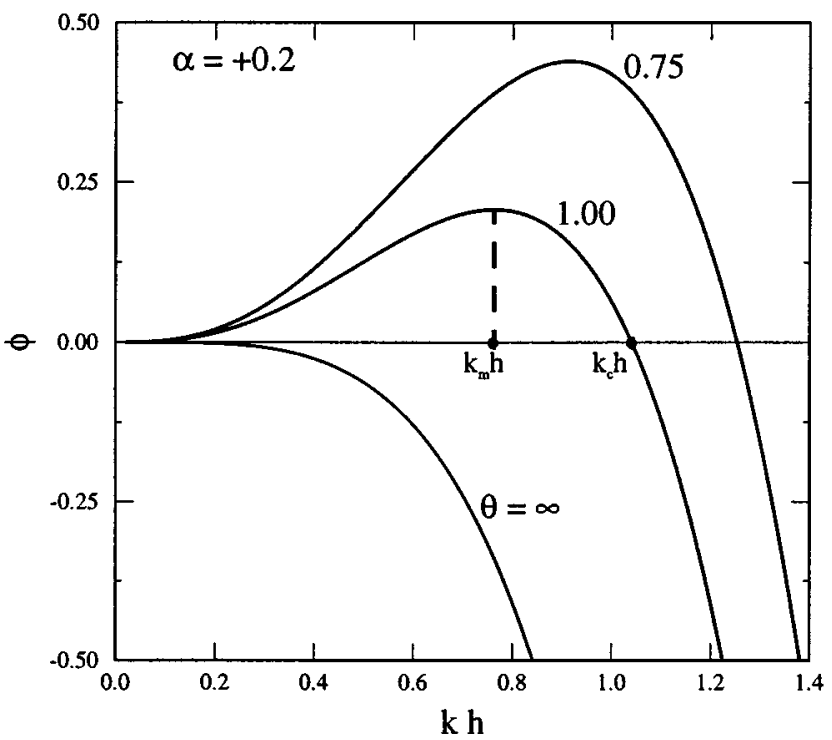

FIG. 2. The dimensionless perturbation amplitude growth rate $\phi$ as a function of the perturbation wave number $k h$ for different values of the normalized interface energy $\theta\left[=\gamma / E_{A} h\left(\epsilon^{*}\right)^{2}\right]$.

number $k h$ for different values of $\theta=\gamma / E_{A} h\left(\epsilon^{*}\right)^{2}$ (at fixed $\alpha)$.

In the absence of a misfit strain $(\theta \rightarrow \infty), \phi_{e}=0$ and $\phi$ is always negative, implying that the flat interface is stable. ${ }^{19}$ On the other hand, for finite $\theta$ (a nonzero misfit strain), the qualitative dependence of the growth rate on the wave number is changed. The dominant terms in the small and large wave number limit can be written as

$$
\begin{array}{ll}
\phi_{c}=-(k h)^{4} \quad \phi_{e} \approx \frac{1}{\theta} \frac{3 \alpha(\alpha+4)}{\left(1-\alpha^{2}\right)}(k h)^{3} \quad \text { for } \quad k h \ll 1 \\
\phi_{c}=-(k h)^{4} \quad \phi_{e} \approx \frac{1}{\theta} \frac{12 \alpha}{(4-\alpha)}(k h)^{3} \quad \text { for } \quad k h \gg 1 .
\end{array}
$$

It is clear that the elastic term dominates the overall growth rate for small wave numbers and the curvature term dominates for large wave numbers. This competition between the elastic and curvature effects dictates the stability of the interface. Therefore, small wave number perturbations grow due to the destabilizing influence of the elastic effects and large wave number perturbations shrink due to the stabilizing influence of curvature.

Figure 2 shows the critical wave number $\left(k_{c} h\right)$ corresponding to the zero growth rate condition $(\phi=0)$. All wave numbers with $k h<k_{c} h$ tend to grow. However, the growth rate is a maximum for a particular wave number $\left(k_{m} h\right)$ and, hence, this is the characteristic wave number which would likely be observed. On increasing the magnitude of the misfit (decreasing $\theta$ ), the general behavior of the instability is not qualitatively changed. Rather, $k_{c} h$ is increased, $k_{m} h$ shifts to higher values, and the perturbation growth rate increases. In other words, the instability wave number and its growth rate both increase with decreasing interface energy $\gamma$ and/or increasing misfit.

We next examine the effect of the elastic mismatch parameter $\alpha$ on interface instability. It is apparent from Eq. (7) 
that the stability condition at small wave numbers is dictated by the sign of $\alpha$. The growth rate, which is controlled by the elastic contribution for small wave numbers, is positive (negative) for $\alpha>0(\alpha<0)$. Figure 3(a) shows the variation of the growth rate $\phi$ as a function of the wave number $(k h)$ for different values of $\alpha$. In the absence of an elastic mismatch $(\alpha=0), \phi$ is always negative implying that the flat interface is stable. This is because, in the absence of an elastic constant mismatch, curvature effects lead to terms which are first order in $(\delta / \lambda)$, whereas contributions due to elastic effects are second order in $(\delta / \lambda)$. However, in the $E_{A}>E_{B}(\alpha>0)$ case, there is a range of wave numbers where the growth rate is positive and consequently the interface is unstable for $k h$ $<k_{c} h$. Therefore, a misfitting layer that is stiffer than the surrounding film is inherently unstable. On the other hand, we observe that for $E_{A}<E_{B}(\alpha<0)$ the growth rate is always negative, and therefore the interface of an elastically compliant misfitting buried layer is stable against shape perturbations. Finally, we present these results in the form of a stability diagram [Fig. 3(b)]. The stability diagram reemphasizes that for all $\alpha \leqslant 0(\alpha>0)$, which corresponds to a compliant (stiff) misfitting buried layer, the flat interface is stable (unstable). In addition, for $\alpha>0$, contours of constant $k_{m} h$ are also shown. Using these contour plots, the maximally unstable wavelength for any $(\alpha, \theta)$ can be estimated.

\section{Multilayer case}

We now examine the stability of multilayer films [see Fig. 1(a)]. When the thickness of the A and B layers is comparable, elastic interactions between them cannot be ignored. We again use the elasticity results of Ref. 16 to predict the spatial variation of the chemical potential and the growth rate of the perturbation to the interface. In this case, we nondimensionalize time $\tau=t \Omega^{2} M \gamma / H^{4}$ and the growth rate $\phi$ $=G h^{4} / \Omega^{2} M \gamma$ using the interlayer spacing $H$. The perturbation growth rate $\phi$ now has an explicit dependence on the relative thickness of the two layers $f(=h / H)$. As before, we partition the growth rate into the curvature and elastic contributions separately as

$$
\phi=\phi_{c}+\phi_{e}=-(k H)^{4}+\phi_{e} .
$$

The dominant terms in the growth rate, for the small wave number limit, is

$$
\phi_{c} \approx-(k H)^{4} \quad \phi_{e} \approx \frac{g(\alpha, f)}{\theta}(k H)^{2} \quad \text { for } k H \ll 1,
$$

where the function $g(\alpha, f)$ is

$$
g(\alpha, f)=12 \alpha(1-\alpha) \frac{\left[(1-2 f)\left(1-3 f+3 f^{2}\right) \alpha^{2}+3\left(1-3 f+3 f^{2}\right) \alpha-2(1-2 f)\right]}{[1-\alpha(1-2 f)]^{2}\left[1-\alpha^{2}\left(1-3 f+3 f^{2}\right)\right]}
$$

Clearly in the small wave number limit, the total growth rate $\phi\left(=\phi_{c}+\phi_{e}\right)$ will be negative provided $g(\alpha, f) \leqslant 0$. Consequently, for a specified layer A relative thickness $(f)$, we can find a range of $\alpha$ over which the flat interface is stable. For volume fractions less than $1 / 2$, the flat interface is unstable for $0<\alpha<\alpha_{c}$ and stable for all other $\alpha$. The critical value $\alpha_{c}$ is related to $f$ by

$$
\alpha_{c}=\frac{3\left(1-3 f+3 f^{2}\right)-\sqrt{\left(1-3 f+3 f^{2}\right)\left(1+5 f-5 f^{2}\right)}}{2(1-2 f)\left(1-3 f+3 f^{2}\right)} .
$$

For a volume fraction of $1 / 2$ (i.e., equal thickness layers), we observe that the flat interface is always stable $\left(\alpha_{c}=0\right)$. On the other hand, for $f>1 / 2$, the interface is unstable for $\alpha_{c}$ $<\alpha<0$ and is stable for all other $\alpha$. Also note that in the limit $f \rightarrow 0$, the stability criteria for the buried layer is recovered.

The effect of the relative layer thicknesses $f(=h / H)$ on the stability criterion is shown graphically in the form of a stability diagram in Fig. 4. For small layer A thicknesses (small $f$ ), the stability condition is essentially unmodified from the buried layer (of phase A) case: i.e., the interface is unstable if the misfitting layer is stiff $(\alpha>0)$ and stable if the misfitting layer is soft $(\alpha<0)$. However, as $f$ increases towards $1 / 2$, the elastic interactions between layers become important and the stability is strongly affected. In addition, it is apparent from Fig. 4 that the stability condition for $(f, \alpha)$ is the same as that for $(1-f,-\alpha)$. For example, we should expect the stability condition pertaining to $f=1 / 3$ and $\alpha=1 / 4$ should be exactly identical to $f=2 / 3$ and $\alpha=-1 / 4$. Clearly, from symmetry arguments, we should expect this result since our definition of layers A and B is arbitrary in the multilayer case. The net result is that a multilayer structure with a compliant minority phase is always stable. If the minority phase is stiff, the stability of the system is dependent on its volume fraction, as per Fig. 4. In general, we see that as we make the thicknesses of the two types of layers more and more similar, the range of $\alpha$ for which instabilities may occur gets smaller and smaller. In the limit that the two types of layers are of exactly the same thickness, the multilayer film is stable for all $\alpha$.

\section{B. Substrate misfit strain}

In the previous case, we assumed that the film was strain balanced with respect to the substrate. By this, we meant that the film was not constrained by the substrate (i.e., if the film was removed from the substrate, it would not undergo any instantaneous strain). We now relax this assumption and consider the case in which the substrate introduces a strain into the film $\epsilon_{x x}=\epsilon^{s}$. This can be thought of in the following way: first consider a free standing film, with or without misfit between the layers. This film has a natural length. We now 


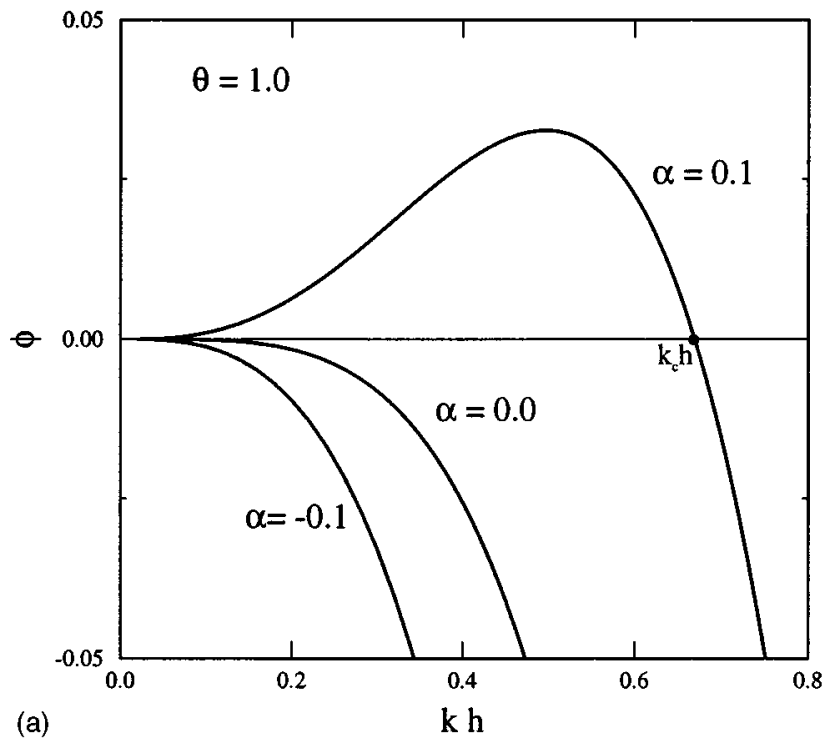

(a)

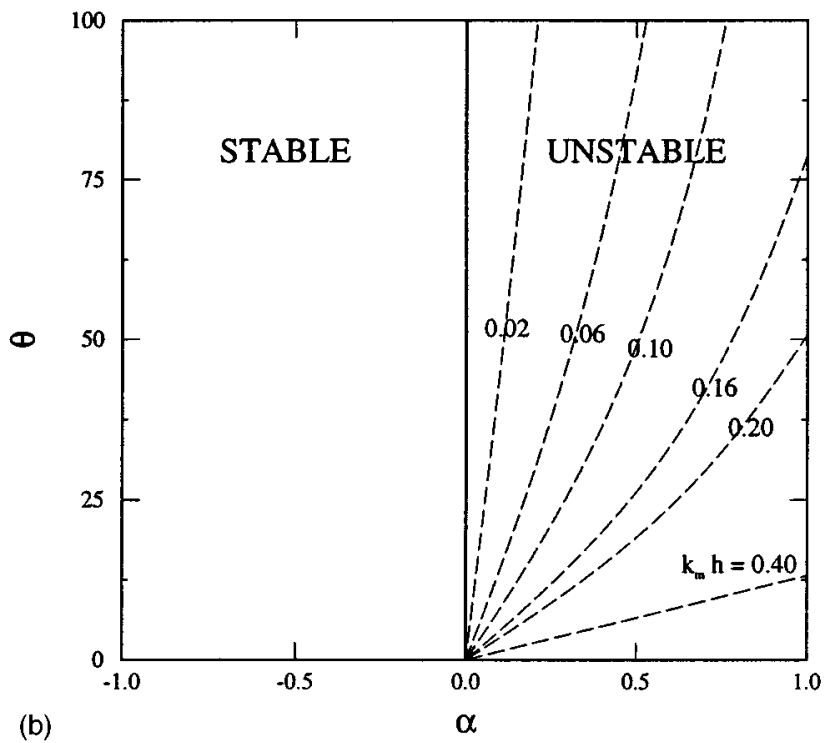

(b)

FIG. 3. (a) The dimensionless perturbation amplitude growth rate $\phi$ for the buried, misfitting layer as a function of the perturbation wave number $k h$ for different values of the elastic mismatch parameter $\alpha$ (for fixed interface energy $\theta$ ). (b) Stability diagram indicating stability of the flat interfaces for all $\alpha \leqslant 0$ and instability of the flat interfaces for all $\alpha>0$. Also shown are contours of constant $k_{m} h$, as a function of the normalized interface energy $\theta$ and the elastic mismatch parameter $\alpha$.

put this film onto the substrate. However, before doing so, we stretch (or compress) the film by some amount, corresponding to a strain $\epsilon_{x x}=\epsilon^{s}$. Such strains can be used to modify the stability of the multilayer structure and can be introduced through heteroepitaxy or a temperature change (providing there is a thermal expansion mismatch between film and substrate). In order to focus on the effects of the substrate misfit strain, we assume here that there is no misfit between the A and B layers of the multilayer film.

\section{Buried layer case}

The substrate misfit strain is imposed in a direction parallel to the unperturbed interface as shown in Fig. 1(b). In this case, it is convenient to redefine the dimensionless inter-

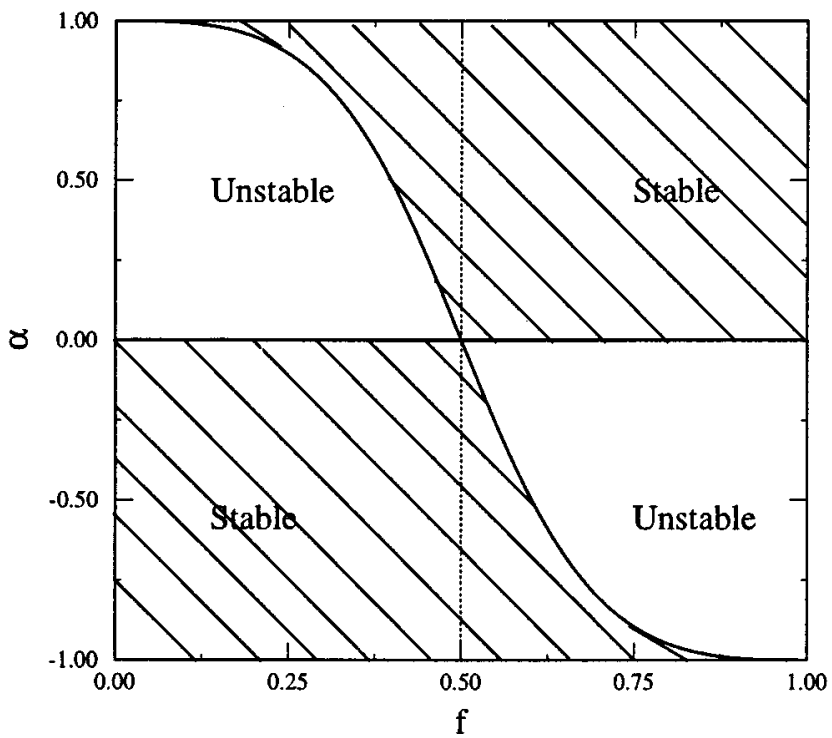

FIG. 4. Stability diagram for the interfaces in a multilayer film (in which the $\mathrm{A}$ and B layers are misfitting with respect to each other) as a function of the relative layer thicknesses $(f=h / H)$ and the elastic mismatch parameter $(\alpha)$. The shaded regions in the diagram indicate conditions under which the flat interfaces are stable. The line demarcating the stable and the unstable regions in the diagram is given by Eq. (10).

face energy as $\theta=\gamma / E_{A} h\left(\epsilon^{s}\right)^{2}$. We initially consider the stability of a buried layer of $\mathrm{A}$ in $\mathrm{B}$. The dominant terms in the growth rate, for the small and large wave number limit, are

$$
\begin{aligned}
& \phi_{c}=-(k h)^{4} \quad \phi_{e} \approx \frac{1}{\theta} \frac{135 \alpha^{2}}{16\left(1-\alpha^{2}\right)}(k h)^{3} \quad \text { for } k h \ll 1 \\
& \phi_{c}=-(k h)^{4} \quad \phi_{e} \approx \frac{1}{\theta} \frac{135 \alpha^{2}}{2\left(16-\alpha^{2}\right)(1+\alpha)}(k h)^{3}
\end{aligned}
$$

for $k h \gg 1$.

It is clear from Eq. (11) that the elastic term dominates the overall growth rate $\phi\left(=\phi_{c}+\phi_{e}\right)$ for small wave numbers and, hence, the substrate misfit destabilizes the interface in this limit. On the other hand, the curvature term dominates the growth rate at large wave numbers and, consequently, the interface is stable for large wave number perturbations. Therefore, small wave number perturbations grow due to the stabilizing influence of the elastic effects and large wave number perturbations shrink due to the stabilizing influence of curvature.

The variation of the growth rate $\phi$ as a function of the wave number $(k h)$ for different values of the modulus mismatch parameter $\alpha$ (and for fixed $\theta$ ) is shown in Fig. 5. In the absence of an elastic mismatch $(\alpha=0), \phi$ is negative (due to the capillarity effect), implying that the flat interface is stable. However, for $\alpha \neq 0$, there is a range of wave numbers $k h<k_{c} h$ where $\phi$ is positive (for both positive and negative $\alpha$ ). This implies that small wave number perturbations grow and large wavelength perturbations shrink for $\alpha \neq 0$. Consequently, as long as the elastic properties of the two phases are different, a flat interface is inherently unstable due to the substrate misfit strain. Although the interface is unstable for all nonzero $\alpha$, the maximally unstable wave number, $k_{m} h$, however, depends on the value and sign of $\alpha$, in addition to its dependence on the normalized interface energy $\theta$. Further 


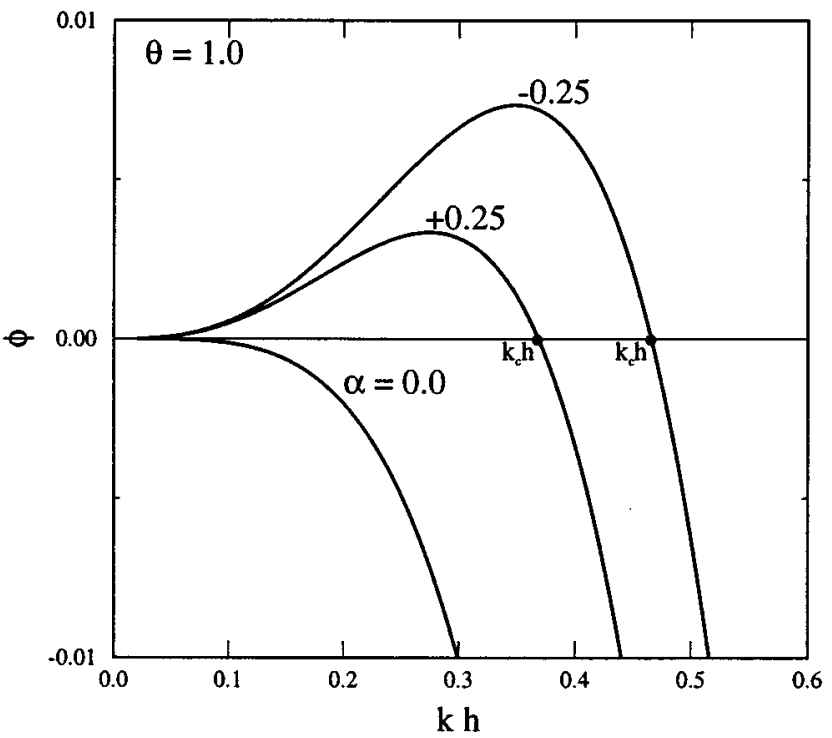

FIG. 5. The dimensionless perturbation amplitude growth rate $\phi$ for the case of substrate misfit and zero interlayer misfit strain as a function of the perturbation wave number $k h$ for different values of the elastic mismatch parameter $\alpha$ (for fixed interface energy $\theta$ ).

analysis ${ }^{16}$ shows that the wave number of the maximally unstable interface perturbation should increase as $|\alpha|$ increases and/or $\theta$ decrease. The results shown here are in stark contrast with the case in which the only misfit is between the buried layer and rest of the film. In that case, the interfaces were only unstable if $\alpha<0$, while in the substrate misfit strain case, the interfaces are unstable for all $\alpha \neq 0$.

\section{Multilayer case}

We now examine the effect of substrate misfit strain on the stability of interfaces in the case of a multilayer film. The modified boundary conditions and the method for obtaining the elastic fields are described in Ref. 16. In the multilayer film case, the perturbation growth rate $\phi$ is an explicit function of the relative thickness of the $\mathrm{A}$ and $\mathrm{B}$ layers $f$ $(=h / H)$, in addition to the other parameters mentioned in the previous section.

Our analysis shows that the interface stability condition derived for the buried layer case is not qualitatively modified upon going to the multilayer case, $f>0$. The interface is always unstable as long as the elastic properties of the two phases are different $(\alpha \neq 0)$, irrespective of the relative layer thicknesses. However, the maximally unstable wavelength is affected by the value of $f$, in addition to $\alpha$ and $\theta$. Figure 6 shows this variation, where the dimensionless maximally unstable wave number $\left(k_{m} H\right)$ is plotted as a function of $\alpha$, for several different values of $f$. In this plot, the normalized interface energy $\theta=\gamma / E_{f} H\left(\epsilon^{s}\right)^{2}$ is kept fixed, where the overall film modulus is $E_{f}=f E_{A}+(1-f) E_{B}$. We observe that at small $|\alpha|$ the instability wave numbers are not significantly altered by changes in the relative thickness of the layers. However, for larger $|\alpha|$, the instability wave number is dependent on these thicknesses. For instance, when $\alpha=$ -0.5 , the instability wave number increases from 0.6 to 0.8 upon an increase in $f$ from 0.25 to 0.5 . This result can be

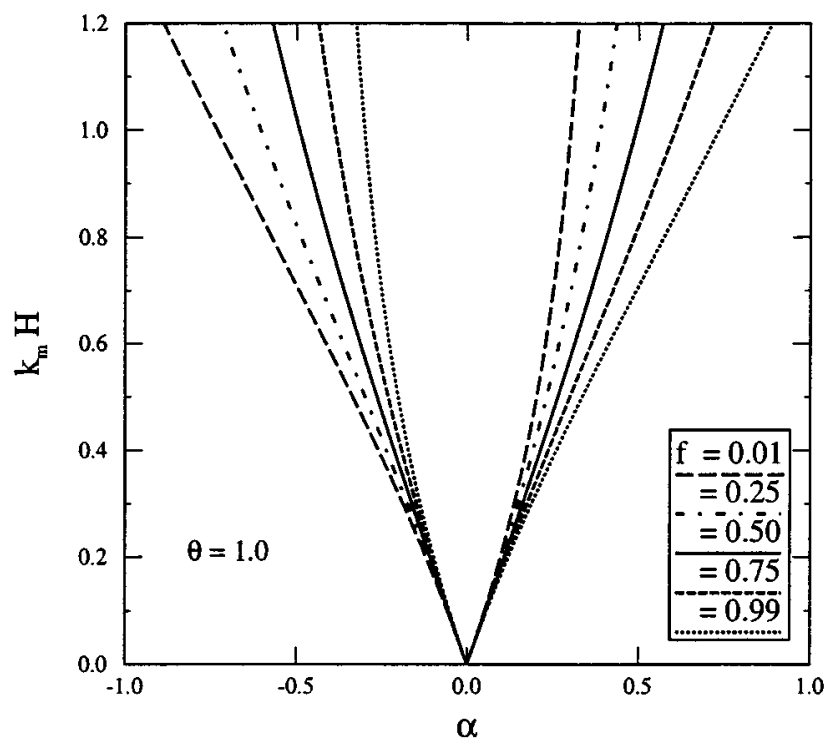

FIG. 6. The maximally unstable wave number $k_{m} H$ for a multilayer film subjected to a substrate misfit strain (no interlayer misfit) as a function of $\alpha$, for several different layer thicknesses $(f=h / H)$. The normalized interface energy $\theta\left[=\gamma / E_{f} H\left(\epsilon^{s}\right)^{2}\right]$ is kept fixed, where $E_{f}$ is the film modulus.

understood as follows. For small A layer thicknesses, the different A layers interact weakly and, therefore, the dominant effect of changing $f$ is to change the overall film modulus. Consequently, if $\theta$ is kept fixed, the instability wave number should not depend on the relative layer thicknesses. However, for large layer A thicknesses, where the interlayer separation $H$ is comparable to the A layer thickness $h$, the elastic interactions are strong. Therefore, the instability wave number is altered despite having taken the film compliance change into account. In addition, as expected $k_{m} h$ is symmetric about $\alpha=0$, provided the thinner layer is defined as layer A. For example, the instability wave number for $(f, \alpha)$ $=(1 / 4,1 / 2)$ is exactly the same as that for $(f, \alpha)=(3 / 4$, $-1 / 2)$

\section{Combined effects of interlayer and substrate misfit strains}

In the previous two sections, we examined the effects of interlayer and substrate misfit strains individually. In this section we consider the combined effects of both. Based on the stability diagram in Fig. 3(b), we expect the interlayer misfit strains can be chosen so as to counteract the destabilizing influence of the substrate misfit. Clearly, this compensation depends on the sign of the mismatch parameter $\alpha$. The flat interface is stable when $\phi \leqslant 0$ for all perturbation wave numbers. For the buried layer case, this condition is satisfied provided

$\alpha\left(\frac{\epsilon^{*}}{\epsilon^{s}}-\frac{1}{(1+\nu)}\right)\left(\frac{\epsilon^{*}}{\epsilon^{s}}-\frac{\alpha(3-4 \nu)}{(1+\nu)[\alpha(1-2 \nu)+2(1-\nu)]}\right) \leqslant 0$,

where $\nu$ is the Poisson ratio, $\epsilon^{*}$ is the dilatational misfit, and $\epsilon^{s}$ is the substrate misfit strain. The balance between the two strains is depicted graphically in the stability diagram in Fig. 7 (a) (setting $\nu=1 / 3$ ). This diagram shows that, for any value 


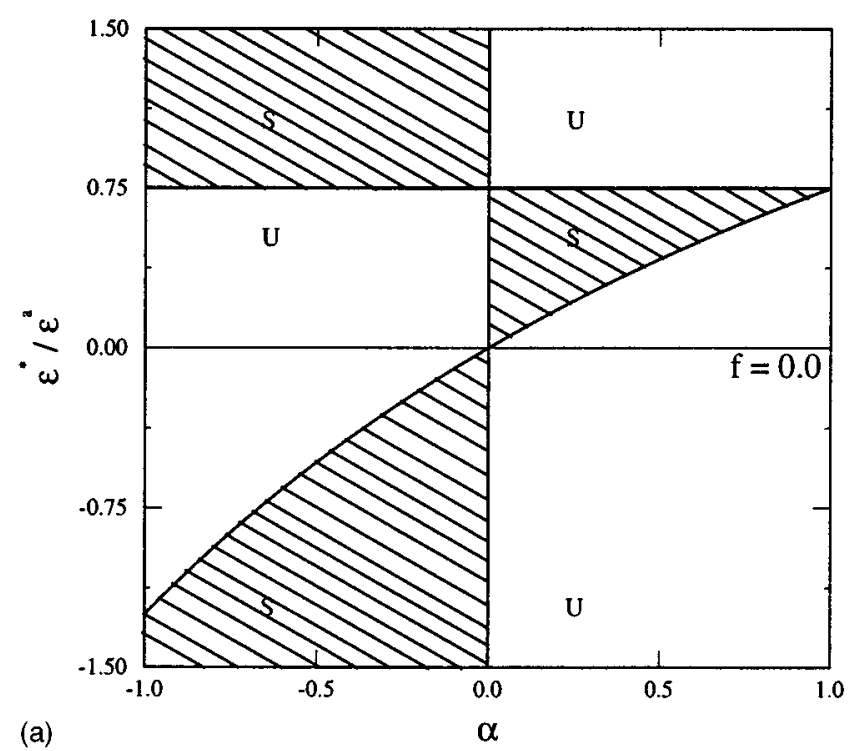

(a)

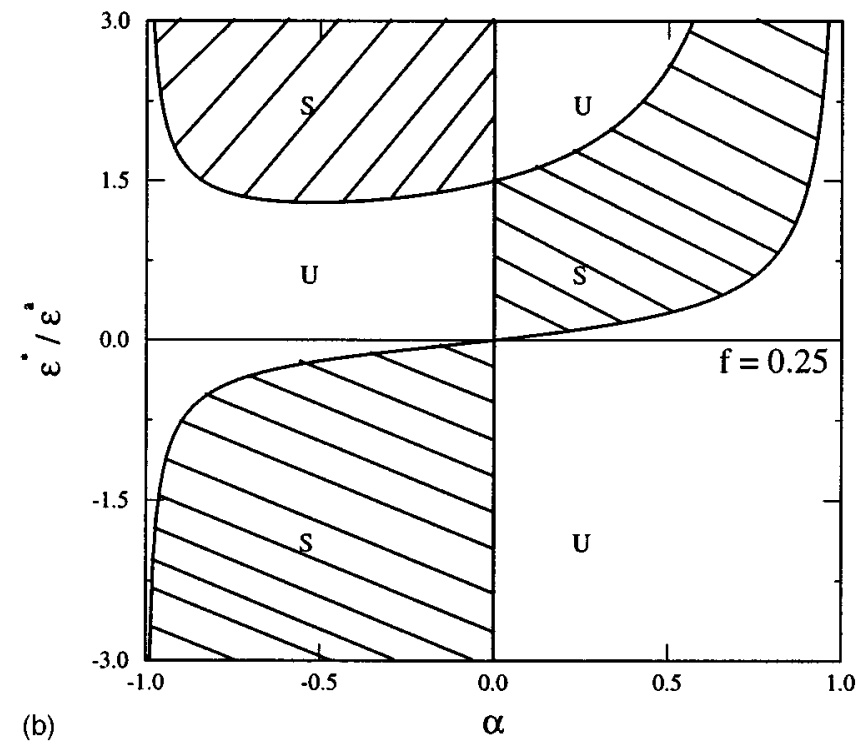

(b)

of the elastic mismatch, there is a range of $\epsilon^{*} / \epsilon^{s}$ for which the flat interface is stable. Therefore, an interlayer misfit strain, of appropriate magnitude, is capable of stabilizing the interface morphology against substrate misfit induced morphological instabilities.

The morphological stability diagram shown in Fig. 7(a) is modified in going from the buried layer to multilayer case because of the elastic interactions between layers. The resultant diagram is, not surprisingly, dependent upon the relative thicknesses of the layers, $f$, as shown in Figs. 7(b) and 7(c). Figure 7(b) shows the stability diagram for $f=1 / 4$, while Fig. 7(c) shows that for the equal layer thickness case, $f$ $=1 / 2$. These results show that increasing the thickness of layer A from zero to one half (at fixed $H$ ) significantly alters the shape (and topology) of the stability diagram. This is especially true near the $\alpha=1$ (rigid A) and $\alpha=-1$ (rigid B) limits. For the special case of equal A and B layer thicknesses $(f=1 / 2)$, the stability diagram is antisymmetric about the $\left(\epsilon^{*} / \epsilon^{s}, \alpha\right)$ origin. Furthermore, for $f>1 / 2$, the stability diagrams will be physically equivalent to the stability

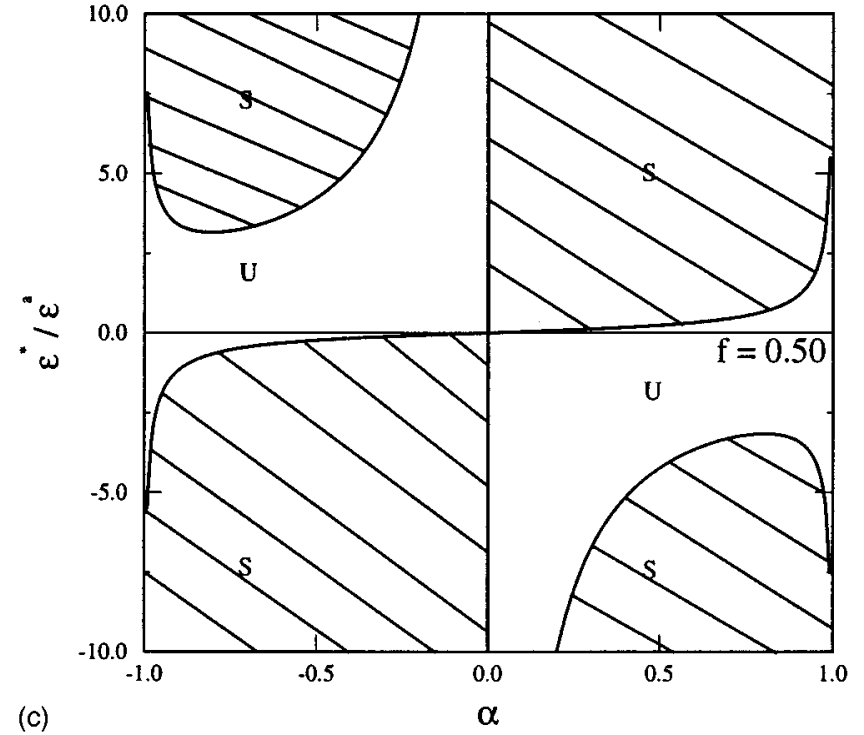

FIG. 7. Stability diagram for the interfaces in a film as a function of $\epsilon^{*} / \epsilon^{s}$ and $\alpha$ for (a) a buried layer ( $f=0$ ), (b) a multilayer film with $f=0.25$, and (c) a multilayer film with $f=0.50$. The shaded regions indicate where the flat interface is stable (denoted by $S$ ) and the unshaded regions indicate regions where the flat interface is unstable due to elastic effects (denoted by $U)$.

diagrams of $f<1 / 2$, provided we label the thinner layer as A.

To summarize, the stability diagrams shown in Fig. 7 predict the conditions under which the flat interface is stable. Such diagrams can be used to provide guidelines for the design of stable, multilayer films.

\section{CONCLUSIONS}

This article shows that misfit stresses can destabilize a multilayer film structure under conditions where interfacial diffusion is significant. These results show that misfit between the layers in the film can destabilize the multilayer structure in cases in which the thinner layer is elastically stiffer than the thicker layer. On the other hand, thin compliant layers stabilize the structure. The rate at which these instabilities develop increase with increasing misfit and decreasing interfacial energy. Even when there is no misfit between the layers, misfit between the multilayer film and substrate can destabilize the interfaces. This type of instability occurs whether the thinner layers are stiffer or more compli- 
ant than the thicker ones. By appropriate choice of the elastic moduli mismatch between layers and relative layer thicknesses, the presence of an interlayer misfit can suppress the instability caused by the substrate misfit. We presented microstructural stability diagrams that can be used to design stable, multilayer films using all of the degrees of freedom commonly available in multilayer film deposition.

We expect these results to be valid for multilayer systems in which the two phases have limited solubility.

\section{ACKNOWLEDGMENTS}

D.J.S. gratefully acknowledges the Division of Materials Science of the Office of Basic Energy Sciences of the United States Department of Energy, Grant No. FG02-88ER45367, for its support of this work. J.M.R. gratefully acknowledges the support of the NSF under Grant No. DMR-9458023.

${ }^{1}$ W. Weisbusch and B. Vinter, Quantum Semiconductor Structures: Fundamentals and Applications (Academic, New York, 1991).

${ }^{2}$ S. T. Chou, K. Y. Cheng, L. J. Chou, and K. C. Hsieh, J. Appl. Phys. 78, 6270 (1995).

${ }^{3}$ Y. Arakawa and H. Sakaki, Appl. Phys. Lett. 40, 939 (1982).
${ }^{4}$ E. P. O'Reilly and A. R. Adams, IEEE J. Quantum Electron. 30, 366 (1994).

${ }^{5}$ L. B. DaSilva, T. W. Barbee, Jr., and R. Cauble, Appl. Opt. 34, 6389 (1995).

${ }^{6}$ D. J. Srolovitz, S. M. Yalisove, and J. C. Bilello, Metall. Trans. A 26, 1805 (1995)

${ }^{7}$ J. M. Millunchick, T. D. Twesten, D. M. Follenstaedt, S. R. Lee, E. D. Jones, Y. Zhang, S. P. Ahrenkiel, and A. Mascarenhas, Appl. Phys. Lett. 70, 1402 (1997).

${ }^{8}$ A. G. Cullis, D. J. Robbins, A. J. Pidduck, and P. W. Smith, J. Cryst. Growth 123, 333 (1992).

${ }^{9}$ A. Ponchet, A. Rocher, J. Y. Emery, C. Starck, and L. Goldstein, J. Appl. Phys. 77, 1977 (1995).

${ }^{10}$ R. J. Asaro and W. A. Tiller, Metall. Trans. 3, 1789 (1972).

${ }^{11}$ M. A. Grinfeld, Sov. Phys. Dokl. 31, 831 (1986).

${ }^{12}$ D. J. Srolovitz, Acta Metall. Mater. 37, 621 (1989).

${ }^{13}$ D. J. Eaglesham and M. Cerullo, Phys. Rev. Lett. 64, 1943 (1990).

${ }^{14}$ W. H. Yang and D. J. Srolovitz, J. Mech. Phys. Solids 42, 1551 (1994).

${ }^{15}$ D. E. Jesson, S. J. Pennycook, J. M. Baribeau, and D. C. Houghton, Phys. Rev. Lett. 71, 1744 (1993).

${ }^{16}$ N. Sridhar, J. M. Rickman, and D. J. Srolovitz, Acta Mater. 45, 2715 (1997).

${ }^{17}$ S. Socrate and D. M. Parks, Acta Metall. Mater. 41, 2185 (1992).

${ }^{18} \mathrm{~J}$. D. Eshelby, in Inelastic Behavior of Solids, edited by M. F. Kanninen et al. (McGraw-Hill, New York, 1970), pp. 77-175.

${ }^{19}$ W. W. Mullins, J. Appl. Phys. 30, 77 (1959). 